\title{
Fiber Optics for Multilayered Optical Memory
}

\author{
Yoshimasa Kawata $^{\dagger}$, Masatoshi Tsuji ${ }^{*}$ and Wataru Inami* \\ (2011 년 9 월 1 일 접수; 2011 년 9 월 9 일 심사완료; 2011 년 9 월 23 일 게재확정)
}

\begin{abstract}
We have developed a compact and high-power mode-locked fiber laser for multilayered optical memory. Fiber lasers have the potential to be compact and stable light sources that can replace bulk solid-state lasers. To generate high-power pulses, we used stretched-pulse mode locking. The average power and pulse width of the output pulse from the fiber laser that we developed were $109 \mathrm{~mW}$ and $2.1 \mathrm{ps}$, respectively. The dispersion of the output pulse was compensated with an external single-mode fiber of $2.5 \mathrm{~m}$ length. The pulse was compressed from 2.1 ps to 93 fs by dispersion compensation. The fiber laser we have developed is possible to use as a light source of multilayered optical memory. We also present a fiber confocal microscope as an alignment-free readout system of multilayered optical memories. The fiber confocal microscope does not require fine pinhole position alignment because the fiber core is used as the point light source and the pinhole, and both of which are always located at the conjugated point. The configuration reduces the required accuracy of pinhole position alignment. With these techniques we can present an allfiber recording and readout system for multilayered memories.
\end{abstract}

Key Words : Optical memory, two-photon process, fiber laser, confocal microscopy

\section{Introduction}

Many techniques have been reported and investigated to realize next-generation terabyte optical data storage applications, such as holographic memories [1,2], near-field recording technology using a solid-immersion lens (SIL) [3], superresolution near-field structure (Super-RENS) technology [4], and three-dimensional (3D) multilayered memories [5-9]. 3D memories can increase the recording capacity by increasing the number of recording layers. The technology of current optical memories, such as optical pickups, servo and tracking systems, and aberration corrections, can be used for 3D memories with some modifications and extensions. 3D multilayered optical memories are one of the most promising techniques for next-generation optical data storage.

$\dagger$ Dept. of Mechanical Engineering, Shizuoka University E-mail : kawata@eng.shizuoka.ac.jp TEL : :+81-53-478-1069
The two photon process is one of the key technologies to realize 3D memories for recording bit data. Ultrashort pulsed lasers are required to generate the two photon process effectively, because the absorption probability of the two photon process is proportional to a squared peak intensity of pulses.

Ti: sapphire lasers are used as one of short-pulse lasers for demonstration of two photon recording. They have many advantages such as high power, high repetition rate, and output of ultrashort pulses. However, they are rather large because they contain many bulk components such as lenses, mirrors, and prisms, and the laser cavity is configured in the rectilinear space. They also require water cooling and they are expensive. As a result, it is difficult to use Ti: sapphire lasers for consumer production.

Fiber lasers that use erbium-doped fiber (EDF) are very promising as ultrashort-pulsed lasers [10]. Because a fiber laser oscillates at $1.56 \curlyvee \mathrm{m}$, the wavelength range of fiber laser is similar to that of a Ti: sapphire laser by doubling frequency with nonlinear crystals. In fiber lasers, the laser cavity comprises of ring fibers and light is resonated in the fiber cavity. Fiber lasers have advantages in comparison with bulk solid-state lasers. First, a fiber laser is very compact, because the fiber cavity 
can be bended and the cavity does not require free space. Second, a fiber laser is very stable, because light is confined in the fiber and there is no necessity of water cooling. By splicing each fiber, coupling loss is much reduced and there is no misalignment of the optical system. Finally, the spacial intensity distribution of the output beam shows circular symmetry. Because light is emitted from a singlemode fiber (SMF), the transverse mode of output beam is $\mathrm{TEM}_{00}$.

The readout system of $3 \mathrm{D}$ optical memories also one of the key issues in the realization of commercially available system. They require a high axial resolution in order to reduce crosstalk between layers. A reflection-type confocal system is the promising configurations for use as the readout system of 3D optical memories because it has a high axial resolution [6]. In confocal system the pinhole that located in front of a detector can pass thourgh the light only reflected and scattered at the focal volume. The pinhole stops the light scattered out of focus.

The confocal system requires fine alignment of the pinhole position. Since the pinhole should be exactly located at the conjugated point of the point light source, it limits the usage of the confocal system for the readout data of 3D optical memories. The confocal system also requires some space because it requires relay lenses.

We have developed a fiber laser as a light source and a confocal readout system with alignment-free feature for 3D memories. The light source is highpower erbium-doped fiber laser using stretched-pulse mode locking [11,12]. By introducing fiber optics in 3D memories, we can achive compact laser light source and readout system.

\section{Development of Fiber Laser as a Femtosecond Pulse Source}

In order to generate short and high-power pulses, we used stretched-pulse mode locking [13].

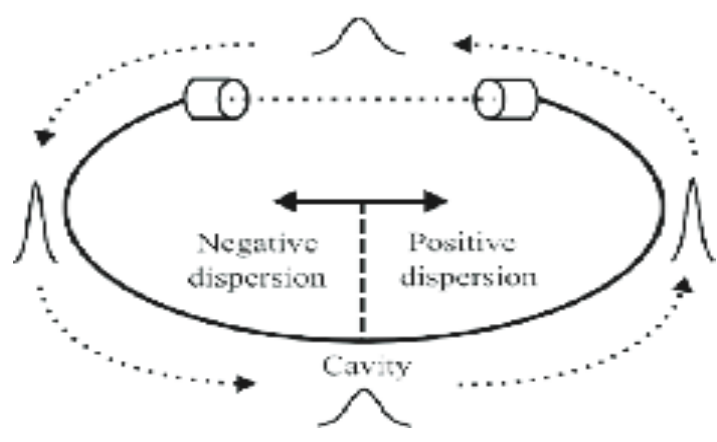

Fig. 1 Concept of stretched-pulse mode locking
In conventional mode locking, output power is limited by a large nonlinearity that causes broadening of pulse width and generation of multiple pulses in the cavity.

Figure 1 shows the concept of stretched-pulse mode locking, which is based on chirped pulse amplification. The ring cavity comprises two segments, which are positive and negative dispersion fibers, in which the pulse is stretched and compressed alternately during its propagation in the cavity. Total cavity dispersion is set to be slightly positive. In the positive dispersion gain fiber, the pulse is stretched and average power increases. Since the pulse peak power is lowered by the stretching, highgain amplification is possible without energy depletion, which is caused by pulse broadening or pulse splitting. The stretched-pulse mode locking can generate a higher average power than that obtained by a conventional method.

Figure 2 shows the optical setup of the fiber laser that we developed. The cavity comprised EDF and SMF. EDF has positive dispersion and SMF has negative dispersion at $1.56 \mu \mathrm{m}$. Two laser diodes (LDs) were used as the pump laser. The wavelength and average power of each LD were $980 \mathrm{~nm}$ and $450 \mathrm{~mW}$, respectively. Pump lights were introduced in a single fiber using a polarization beam combiner (PBC) and coupled into the ring cavity through the wavelength division multiplexing (WDM) coupler. Each fiber was spliced and the splicing loss was negligible.

We measured the spectrum and pulse train of the monitor coupler using a spectrum analyzer and an oscilloscope, respectively. A polarization beam splitter (PBS) operated as the output port.

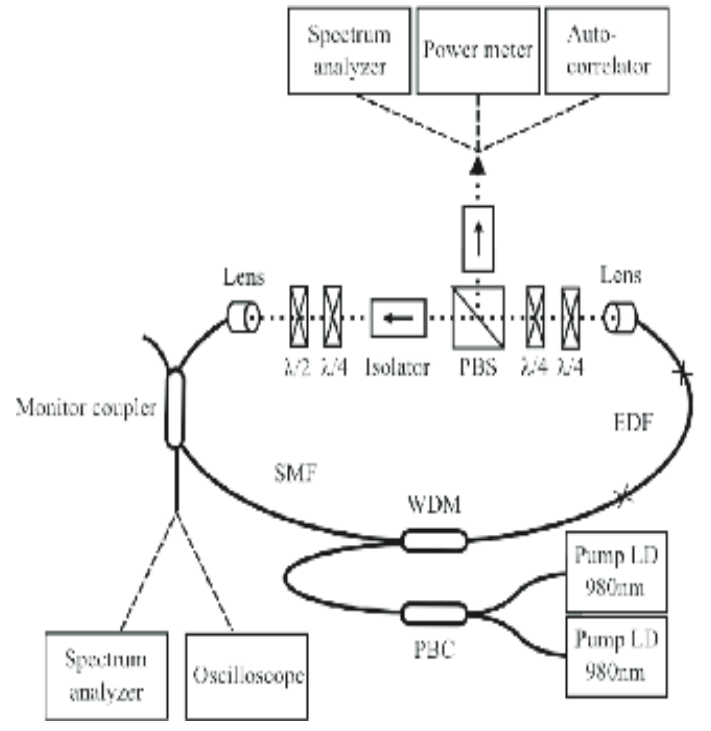

Fig. 2 Optical setup of developed fiber laser. 


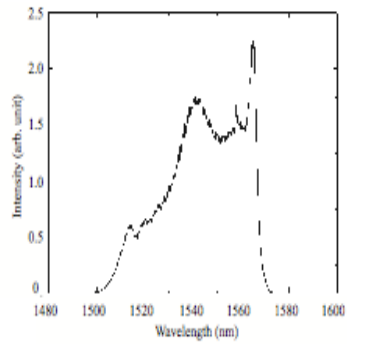

(a)

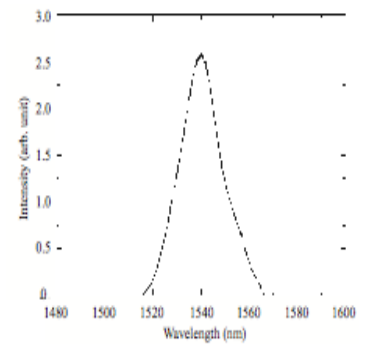

(b)
Fig. 3 Spectra of output pulse from developed fiber laser: (a) spectrum of output pulse from PBS and (b) spectrum of output pulse from monitor coupler.

We also measured the spectrum, average power, and temporal waveform of output beam using a spectrum analyzer, a power meter, and an autocorrelator, respectively.

Figure 3(a) shows the spectrum of the output pulse from the PBS. The spectrum bandwidth was broadened by amplification at EDF. Figure 3(b) shows the spectrum of the output pulse from the monitor coupler. We can observe the well-defined spectrum with Gaussian distribution, as shown in Fig. 3(b). The center wavelength of the spectrum is about $1540 \mathrm{~nm}$. We succeeded in operating with an average power of $109 \mathrm{~mW}$ and high-power pulse generation. The high-power output was achieved by stretched-pulse mode locking.

Figure 4 shows the pulse train measured at the monitor coupler. The time interval of pulse trains was $17.4 \mathrm{~ns}$, and the repetition rate was $57.5 \mathrm{MHz}$. The pulse energy was calculated as $1.9 \mathrm{~nJ}$ from the average power and repetition rate. The output pulse energy of commercially available fiber oscillators is less than $1.2 \mathrm{~nJ}$, so we have succeeded to develop a high-power laser. The pulse shape was well defined and the pulse width was 2.1 ps. In order to increase the probability of the two photon process, the pulse width should be decreased.

In order to decrease the pulse width, we performed dispersion compensation [14]. The output pulse from PBS showed positive chirping. The positively chirped pulse can be compressed to increase peak power during propagation in the negative dispersion components. In this study, we used SMF for dispersion compensation, because SMF is cheap and practical. Since the compression method does not require bulk components such as prisms and a pair of gratings, it should be compact.

The average power at SMF end was $90 \mathrm{~mW}$. Figure 5 shows the temporal waveform of the output pulse after SMF. This waveform was the autocorrelation

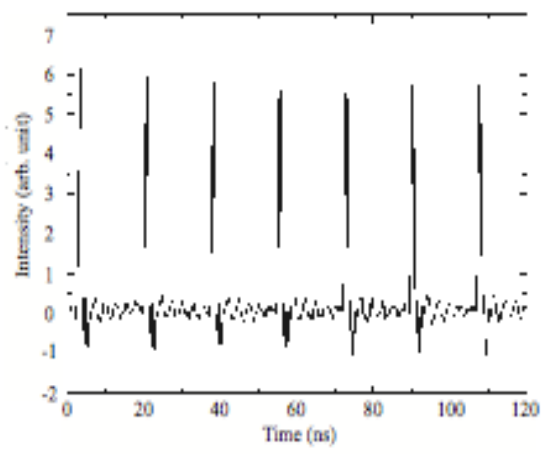

Fig. 4 Pulse train measured at monitor coupler

trace and it was monitored using a two-photon absorption-type autocorrelator (Femtochrome Research, FR-103PD). The autocorrelation trace was composed of three peaks. From the trace, the temporal waveform of the output pulse was presumed to be composed of two peaks. We assumed that each peak in the waveform was of the sech2 shape. By the fitting process of autocorrelation of the temporal waveform to the measured waveform shown in Fig. 5, we derived that the pulse width was $93 \mathrm{fs}$ and the peak power was $5.3 \mathrm{~kW}$.

We believe that the peak power is sufficient to generate the two photon process, because Shiono et al. demonstrated two-photon recording with a peak power of $18 \mathrm{~W}$ [15]. In their experiments, the wavelength was $0.785 \mu \mathrm{m}$ and cis-1,2-dicyano-1,2bis (2,4,5-trimethyl-3-thienyl)ethane was used as the recording material. Kawata et al. also demonstrated the two-photon recording with pulses of which the peak power was $1.1 \mathrm{~kW}$ [16]. They also used light of $780 \mathrm{~nm}$ wavelength. The laser has sufficient peak power, even if we take into account the energy loss due to second harmonic generation (SHG) conversion, which is typically a few tens percent efficiency.

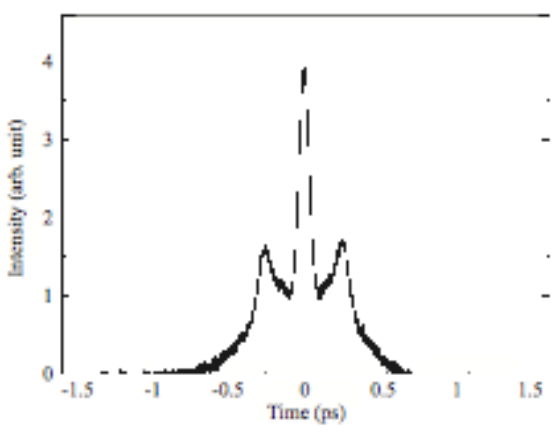

Fig. 5 Temporal waveform of output pulse after SMF 
We carried out an experiment of SHG conversion using the fiber laser that we have developed with a commercial periodically poled lithium niobate (PPLN) (Stratphase). We obtained a SHG conversion efficiency of $18 \%$. We expect to obtain a higher SHG conversion efficiency by the optimization of the focal lens. Moutzouris et al. also demonstrated SHG conversion using 80 fs pulses centered at $1.55 \mu \mathrm{m}$ using poled period $\mathrm{MgO}$-doped $\mathrm{LiNbO}_{3}$ crystals [17]. They obtained a SHG conversion efficiency of $40 \%$. Using these nonlinear crystals, we expect to obtain the peak power sufficient for two photon recording. The fiber laser we have developed is a good candidate as a light source of 3D optical memory.

\section{Fiber Confocal Readout System with Alignment Free Feature}

Figure 6 shows the basic system configuration of the fiber confocal microscope. A fiber coupler is used as the basis of the fiber confocal microscope. The readout laser and the detector are connected with ports 1 and 2 of the fiber coupler, respectively. The laser light propagates into the fiber and reaches the multilayered medium. The signal from the multilayered medium is detected through the fiber coupler.

The fiber confocal microscope increases the noise level in comparison with a conventional confocal system, because the light propagating inside the fiber is reflected at the fiber end at port 3 and the fiber coupler. This reflected light increases the background noise level. Since the reflected light from each recording layer is reasonably small, it should increase the signal-to-noise ratio (SNR) by reducing the level of reflection from the fiber end and the fiber coupler.

We introduced two noise reduction methods into the fiber confocal microscope. First, to reduce the level of reflection at the fiber end, we used an angled

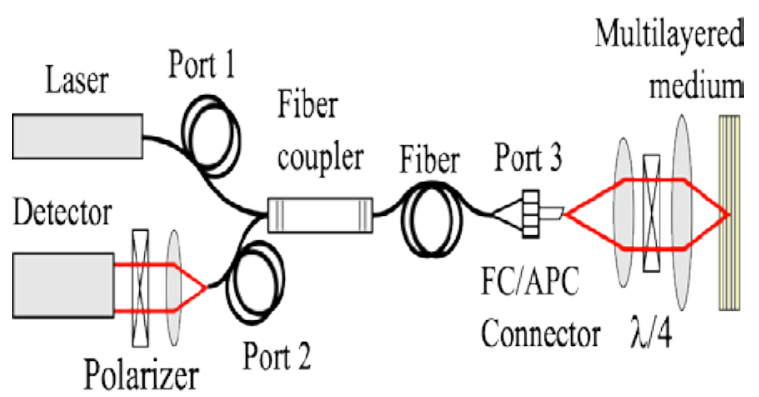

Fig 6 Basic configuration of fiber confocal microscope physical contact (APC) connector at the fiber end. The end face of the APC connector was cut with an angle slanted toward the optical axis. The reflected light at the fiber end did not propagate back in the fiber core. We reduced the reflected light to less than $60 \mathrm{~dB}$.

Second, the signal and the reflection light were separated by controlling the polarization state. We inserted a quarter-wave plate (QWP) and a polarizer into the light path. The linear polarization state of the signal was rotated $90^{\circ}$ because it passed through the QWP twice. The reflected light at the fiber end or the coupler did not pass through the QWP; thus, they maintained the polarization state. The reflection light at the fiber end and the fiber coupler was rejected through the polarizer.

A helium-neon (He-Ne) laser of $633 \mathrm{~nm}$ wavelength was used for readout. The light was focused onto the single-mode fiber (SMF) end and passed through the fiber coupler. The light emitted by the fiber was collimated using an objective of 0.65 numerical aperture (NA) and focused onto the multilayered medium using an oil-immersion objective of 1.3 NA. The signal reflected from the focal spot was focused at the fiber end with the same optics. The signal was separated using the fiber coupler and detected with a photomultiplier tube (PMT). The branch ratio of the fiber coupler was 50:50. The SMF model we used was manufactured by SM600, Fibercore Ltd. The multilayered medium was placed on an XYZ-axis automatic stage. We verified the axial resolution of the fiber confocal microscope using a multilayered sample. The multilayered sample was fabricated with highrefractive-index (HRI) layers and buffer layers. The top was the cover layer, and the bottom was a glass substrate. The refractive indexes of the HRI layers, the buffer layers, and the cover layer were 1.53, 1.466, and 1.518 at a wavelength of $633 \mathrm{~nm}$, respectively. The thicknesses of the HRI layers, the buffer layers, and the cover layer were 1, 10, and 5 $\mu \mathrm{m}$, respectively. The medium had five HRI layers. The HRI layers were prepared with poly(methyl methacrylate) (PMMA). The buffer layers were prepared with butyl acrylate and acrylic acid, and the cover layer was prepared with poly(vinyl alcohol) (PVA). Figure 7(a) shows the axial response of the multilayered sample. We could observe the reflection peaks of the HRI layers. The rightmost peak corresponded to the interface between the cover layer and the buffer layer. The five center peaks indicated the HRI and buffer layer interfaces, and the leftmost peak represented the reflection from the interface between the buffer layer and the glass substrate. Figure 7(b) shows the magnified image of the first HRI layer peak shown in Fig. 7(a). The full width at half maximum (FWHM) of this peak was 
$900 \mathrm{~nm}$. Therefore, we considered that the axial resolution of the fiber confocal microscope was 900 nm. Figure 7(c) shows the axial response of the same sample before the introduction of the two noise reduction methods. The background noise level was higher and fluctuated as shown in Fig. 7(c). It is difficult to distinguish the interface between the buffer layer and the substrate. We succeeded in improving the SNR of the fiber confocal microscope to a level enabling the use of the microscope as the

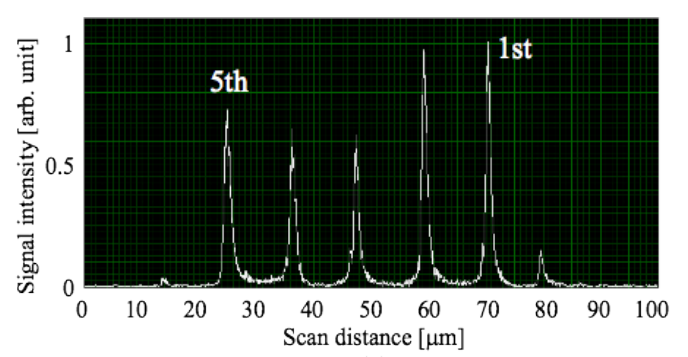

(a)

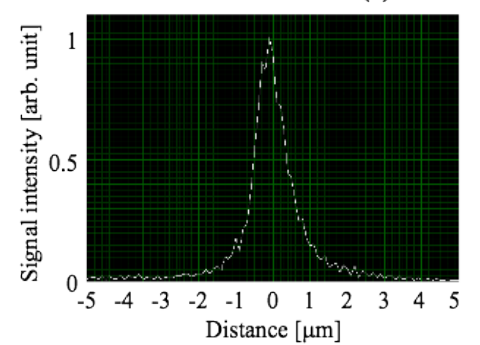

(b)

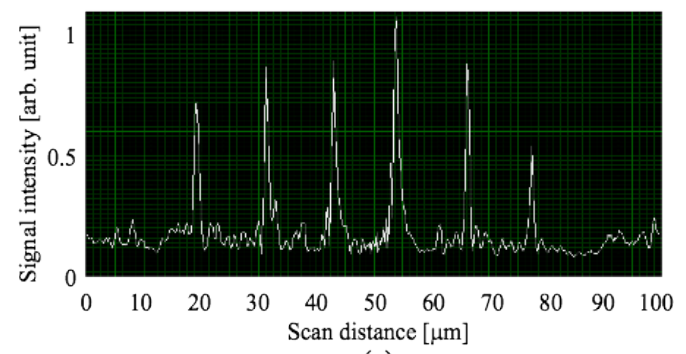

(c)

Fig. 7 Axial response of multilayered sample: (a) axial response with noise reduction methods, (b) magnified image of first peak shown in(a), and (c) axial response without noise reduction methods.

readout system of multilayered memories.

We recorded bit data into the multilayered recording medium and read them out using the fiber confocal microscope. We used the same optical setup for multilayer recording and readout as that shown in Fig. 6. A mode-locked Ti:sapphire laser was used to demonstrate the multilayer recording. The wavelength, pulse width, and repetition rate of the Ti:sapphire laser were $780 \mathrm{~nm}, 80 \mathrm{fs}$, and $80 \mathrm{MHz}$, respectively. The laser pulse was focused onto the multilayered recording medium with an objective of 1.3 NA. A neutral density (ND) filter was used for adjusting the recording power. The exposure time was controlled by a mechanical shutter. The minimum shutter exposure time was $8 \mathrm{~ms}$.

The multilayered medium consisted of ten recording layers and eleven buffer layers. The top was a PVA cover layer, and the bottom was a poly(ethylene terephthalate) (PET) substrate. The thickness of the recording layers, the buffer layers, and the cover layer were 1,5 ,and $1 \mu \mathrm{m}$, respectively.

The recording layers were prepared with PMMA. The photochromic material diarylethene (DE) was doped to PMMA. The concentration of DE as the recording material was $10 \%$ by weight.

The composition of DE was cis-1,2-dicyano-1,2bis(2,4,5-trimethyl-3-thienyl)ethene.

The multilayered recording medium was fabricated using a lamination process [8].

Figure 8 shows the multilayer recording and readout results obtained using the fiber confocal microscope.

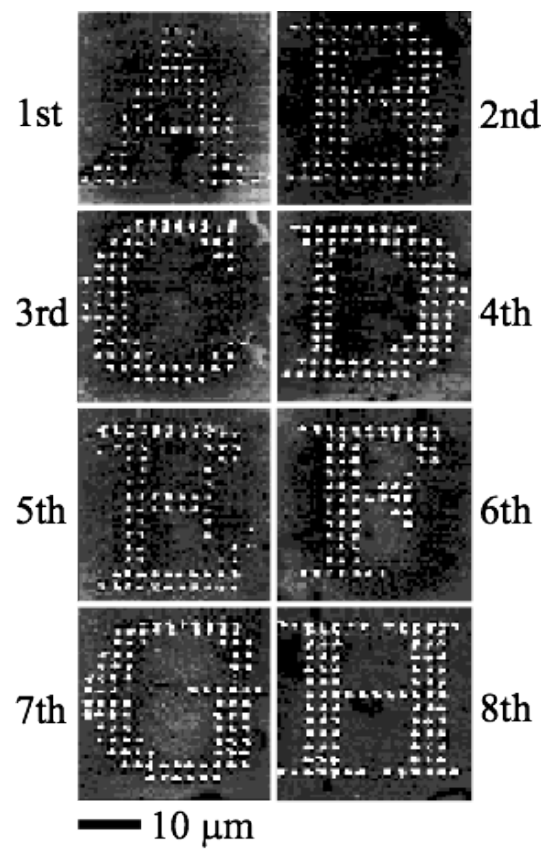

Fig. 8 Multilayer recording and readout results using fiber confocal microscope.

The bright and dark parts indicate the signal from the bit data and the reflection from the recording layer interface, respectively. We could achieve the eightlayer recording and readout. The alphabet patterns of each layer were recorded with the sequence of bit data. These recorded bit data were formed by a two-photon absorption (TPA) process. The bit interval was $2 \mu \mathrm{m}$. The average power and the exposure time were $61 \mathrm{~mW}$ and $8 \mathrm{~ms}$, respectively. The low contrast shown in the first layer was caused 
by the lens aberration.

Figure 9(a) shows the axial response of the multilayered recording medium before recording. All recording layers could be detected using the fiber confocal microscope. The highest peak represented the reflection at the buffer layer and PET substrate interface. Figure 9(b) shows the axial cross section of the multilayered recording medium after recording. The bottom white line represents the interface of the buffer layer and the PET substrate, and the top line represents the interface of the cover layer and the buffer layer. The ten lines between the cover layer and the PET substrate interfaces indicate the recording layer interfaces. The recorded bits could be detected in the recording layers.

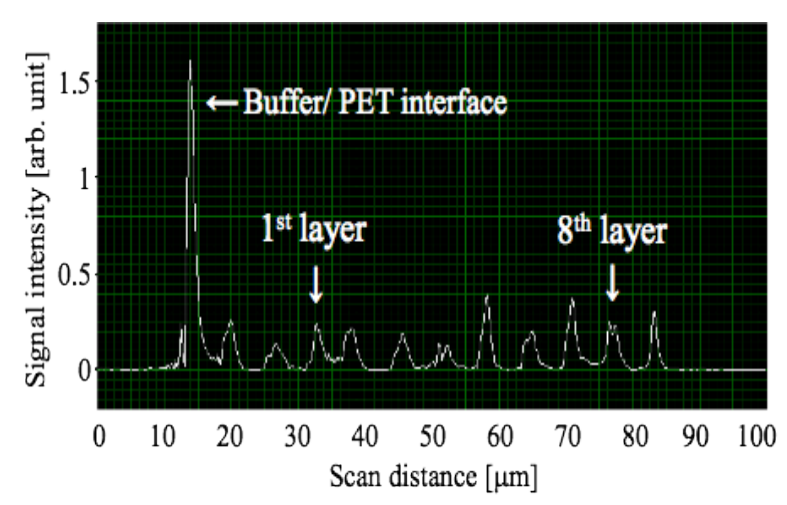

(a)

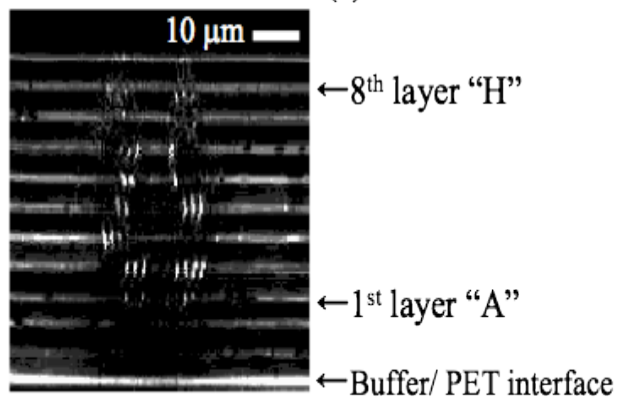

(b)

Fig. 9 (a) Axial response of multilayered recording medium before recording. (b) Axial cross section of multilayered recording medium after recording.

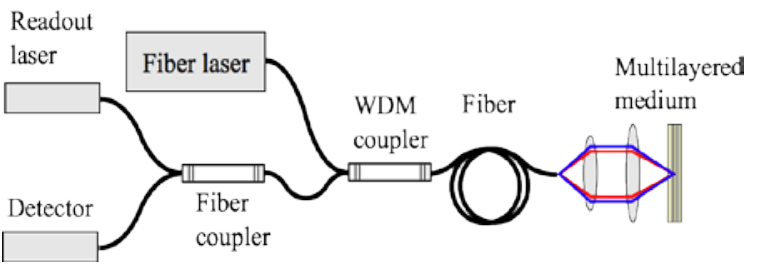

Fig. 10 Concept of all-fiber recording and readout system.

\section{Proposal of All-Fiber Recording and Readout System}

We propose an all-fiber recording and readout system for multilayered optical memories. Figure 10 shows the concept of the all-fiber recording and readout system. The all-fiber system consists of an ultrashort-pulse fiber laser, a readout laser, and a readout detector. These optical systems are assembled using a single fiber with a wavelength division multiplex (WDM) coupler. The WDM coupler serves as a dichroic mirror. This system has the potential of an all-fiber compact system. Moreover, this system has a flexible configuration.

\section{Conclusion}

We developed a compact and high-power modelocked fiber laser for 3D optical memory. Fiber lasers have the potential to be compact and stable light sources that can replace bulk solid-state lasers. The average power, repetition rate, and pulse width of the fiber laser that we developed were $109 \mathrm{~mW}$, $57.5 \mathrm{MHz}$, and $2.1 \mathrm{ps}$, respectively. In order to increase the probability of the two photon process, we decreased the pulse width from 2.1 ps to 93 fs by pulse compression using a SMF. The average power at the SMF end was $90 \mathrm{~mW}$ and the peak power was $5.3 \mathrm{~kW}$. We need SHG conversion for recording to highly sensitive materials of the two photon process. The fiber laser is a good candidate as a light source of 3D optical memory.

We also developed a fiber confocal microscope as a readout system of $3 \mathrm{D}$ memories. To increase the SNR, we introduced an APC connector and a polarization control technique. The background noise level was reduced to less than $60 \mathrm{~dB}$. We demonstrated that the axial resolution of the fiber confocal microscope was $900 \mathrm{~nm}$.

We also demonstrated the reading of bit data from a multilayered recording medium by using the fiber confocal microscope. The fiber confocal microscope could read out the bit data without crosstalk. From the obtained results, the fiber confocal microscope was considered to be a feasible as compact readout system of 3D multilayered optical memories.

We proposed an all-fiber recording and readout system for multilayered memories. The system was made compact by a combination of a fiber confocal readout system and an ultrashort-pulse fiber laser as a recording light source. The all-fiber system that we proposed is promising as a 3D multilayered optical memory system. 


\section{Acknowledgement}

We are very grateful to LINTEC Corporation for providing us with the multilayered sample for the axial resolution measurement and the multilayered recording medium for the multilayer recordin

\section{References}

[1] L. Dhar, A. Hale, H. E. Katz, M. L. Schilling, M. G. Schnoes, and F. C. Schilling, 1999, Opt. Lett., Vol.24, pp 487

[2] H. Horimai, X. Tan, and J. Li, 2005, Appl. Opt., Vol.44, pp. 2575

[3] M. Shinoda, K. Saito, T. Ishimoto, T. Ito, A. Nakaoki, M. Yamamoto, O. Maeda, T. Hashizu, T. Asano, K. Aga, K. Takagi, and M. Tazoe, 2006, Jpn. J. Appl. Phys., Vol. 45, pp. 1321

[4] J. Tominaga, H. Fuji, A. Sato, T. Nakano, T. Fukaya, and N. Atoda, 1998, Jpn. J. Appl. Phys., Vol. 37, L1323

[5] S. Kawata and Y. Kawata, 2000, Chem. Rev., Vol. 100, pp. 1777

[6] M. Ishikawa, Y. Kawata, C. Egami, O. Sugihara, N. Okamoto, M. Tsuchimori, and O. Watanabe, 1998, Opt. Lett.,, Vol.23, pp. 1781

[7] M. Gu and D. Day, 1999, Opt. Lett., Vol. 24, pp. 288

[8] M. Miyamoto, M. Nakano, M. Nakabayashi, S. Miyata, and Y. Kawata, 2005, Appl. Opt., Vol. 45, pp. 8424

[9] M. Nakano, T. Kooriya, T. Kuragaito, C. Egami, Y. Kawata, M. Tsuchimori, and O. Watanabe, 2004, Appl. Phys. Lett., Vol. 82, pp. 176

[10] K. Tamura, E. P. Ippen, H. A. Haus, and L. E. Nelson, 1993, Opt. Lett., Vol. 18, pp. 1080

[11] K. Tamura, C. R. Doerr, L. E. Nelson, H. A. Haus, and E. P. Ippen, Opt. Lett., Vol. 19, pp. 46

[12] N. Nishizawa, Y. Chen, P. Hsiung, E. P. Ippen, and J. G. Fujimoto, 2004, Opt. Lett., Vol. 29, pp. 2846

[13] M. Tsuji, N. Nishizawa, and Y. Kawata,, 2008, Jpn. J. Appl. Phys., Vol. 47 , pp. 5797

[14] J. Takayanagi, N. Nishizawa, H. Nagai, M. Yoshida, and T. Goto, 2005, IEEE Photonics Technol. Lett., Vol. 17, pp. 37

[15] T. Shiono, T. Itoh, and S. Nishino, 2005, Jpn. J. Appl. Phys. Vol.44 , pp. 3559

[16] S. Kawata, A. Toriumi, and D. Yasumatsu, presented at Focus on Microscopy, 12th Int. Conf. 3D Image Processing in Microscopy/ 11th Int. Conf. Confocal Microscopy, 1999

[17] K. Moutzouris, F. Sotier, F. Adler, and A. Leitenstorfer, 2006, Vol.14, Opt. Express, pp. 1905 